\title{
Precision RENORM Tensor-Pomeron Cross Sections
}

\author{
Konstantin Goulianos ${ }^{1, a}$ \\ ${ }^{1}$ The Rockefeller University, 1230 York Avenue, New York, NY 10065-6399, USA
}

\begin{abstract}
Precision predictions of soft and hard diffraction, elastic, and total protonproton cross sections, based on a Regge-theory inspired tensor-Pomeron implementation of the RENORM model, are compared to TOTEM, ATLAS, and CMS results at the LHC.
\end{abstract}

\section{Introduction}

In DIS-2015 (Spring 2015) we summarized [1] the pre-LHC predictions of the total, elastic and totalinelastic, as well as the single diffractive (SD) and double diffractive (DD) components of the protonproton cross section at high energies, based on the RENORM / MBR model [2]. We compared the measurements of the SD and DD cross sections from the Tevatron and the LHC with the predictions of the model and found excellent agreement. Good agreement was also observed between the model predictions and the total, elastic, and total inelastic cross sections obtained at the Tevatron at $\sqrt{s}=$ $1.8 \mathrm{TeV}$, and at the LHC at $\sqrt{s}=7$ and $8 \mathrm{TeV}$.

The confirmation of the predictions of all the above cross sections at the Tevatron and the LHC up to $\sqrt{s}=8 \mathrm{TeV}$ prompted a calculation/extrapolation to $\sqrt{s}=13 \mathrm{TeV}$, the nominal foreseen collidingbeam energy at the LHC for Summer 2015. For $\sigma_{\text {tot }}, \sigma_{\text {el }}$ and $\sigma_{\text {inel }}$, we predicted $108 \mathrm{mb}, 32 \mathrm{mb}$, and $77 \mathrm{mb}$, respectively, with uncertainties of $\sim 11 \%$ in all cases, mainly due to the uncertainty in the energy-squared scale parameter $s_{0}$ of the model.

In Summer 2015 we updated the value of $s_{0}$ to a more precise one based on a tensor glueball interpretation of the Axial Field Spectrometer (AFS) exclusive charged di-pion data [3-5]. This change in RENORM / NBR decreases the uncertainties in the predictions of the total, elastic, and total-inelastic cross sections to less than $2 \%$ from Tevatron to LHC energies, with little or no effect on the mean values. The predictions were compared with measurements by ATLAS at $\sqrt{s}=7 \mathrm{TeV}$ and by TOTEM at $\sqrt{s}=7$ and $8 \mathrm{TeV}$ at Moriond QCD in March 2016 [6] and found to be in good agreement.

This is an update of [6] that includes verbatim certain sections and describes new measurements of the total inelastic cross section at $\sqrt{s}=13 \mathrm{TeV}$ by ATLAS and CMS at DIS-2016 (April 2016) by Trzebinski ATLAS) and Van Haevermaet (CMS) [7]. There are disagreements among the measurements themselves and the RENORM predictions at the $\sim 2$ sigma level. In the present paper, we discuss these findings in an attempt to understand the origin of the observed discrepancies.

\section{RENORM cross sections}

The total, elastic, and total-inelastic cross sections in the RENORM / NBR model depend on the value of the energy-squared scale parameter, $s_{0}$. Quoting verbatim from Ref. [1],

\footnotetext{
a e-mail: dino@rockefeller.edu
} 
"The total cross section $\left(\sigma_{\text {tot }}\right)$ is expressed as [8]

$$
\begin{array}{lcl}
\sigma_{\text {tot }}^{p^{ \pm} p}= & 16.79 s^{0.104}+60.81 s^{-0.32} \mp 31.68 s^{-0.54} & \text { for } \sqrt{s} \leq 1.8 \mathrm{TeV}, \\
\sigma_{\text {tot }}^{p^{ \pm} p}= & \sigma_{\text {tot }}^{\mathrm{CDF}}+\frac{\pi}{s_{0}}\left[\left(\ln \frac{s}{s_{F}}\right)^{2}-\left(\ln \frac{s^{\mathrm{CDF}}}{s_{F}}\right)^{2}\right] & \text { for } \sqrt{s} \geq 1.8 \mathrm{TeV},
\end{array}
$$

where $s_{0}$ and $s_{F}$ are the energy and (Pomeron flux) saturation scales, $s_{0}=3.7 \pm 1.5 \mathrm{GeV}^{2}$ and $\sqrt{s}_{F}=22 \mathrm{GeV}$, respectively. For $\sqrt{s} \leq 1.8 \mathrm{TeV}$, where there are Reggeon contributions, we use the global fit expression [9], while for $\sqrt{s} \geq 1.8 \mathrm{TeV}$, where Reggeon contributions are negligible, we employ the Froissart-Martin formula [10-12]. The two expressions are smoothly matched at $\sqrt{s} \approx 1.8$ $\mathrm{TeV}$. The $\sigma_{\text {el }}$ for $\sqrt{s} \leq 1.8 \mathrm{TeV}$ is obtained from the global fit [9], while for $1.8<\sqrt{s} \leq 50 \mathrm{TeV}$ we use an extrapolation of the global-fit ratio of $\sigma_{\text {el }} / \sigma_{\text {tot }}$, which is slowly varying with $\sqrt{s}$, multiplied by $\sigma_{\text {tot }}$. The total non-diffractive cross section is given by $\sigma_{\mathrm{ND}}=\left(\sigma_{\mathrm{tot}}-\sigma_{\mathrm{el}}\right)-\left(2 \sigma_{\mathrm{SD}}+\sigma_{\mathrm{DD}}+\sigma_{\mathrm{CD}}\right) . "$

\subsection{Tensor-Pomeron predictions}

The partial wave analysis of the AFS exclusive $\pi^{ \pm}$data [5], performed in terms of a fit with a model with S-wave and D-wave amplitudes as a function of the di-pion mass up to $2.3 \mathrm{GeV}$, leads to the results presented in Fig. 1.

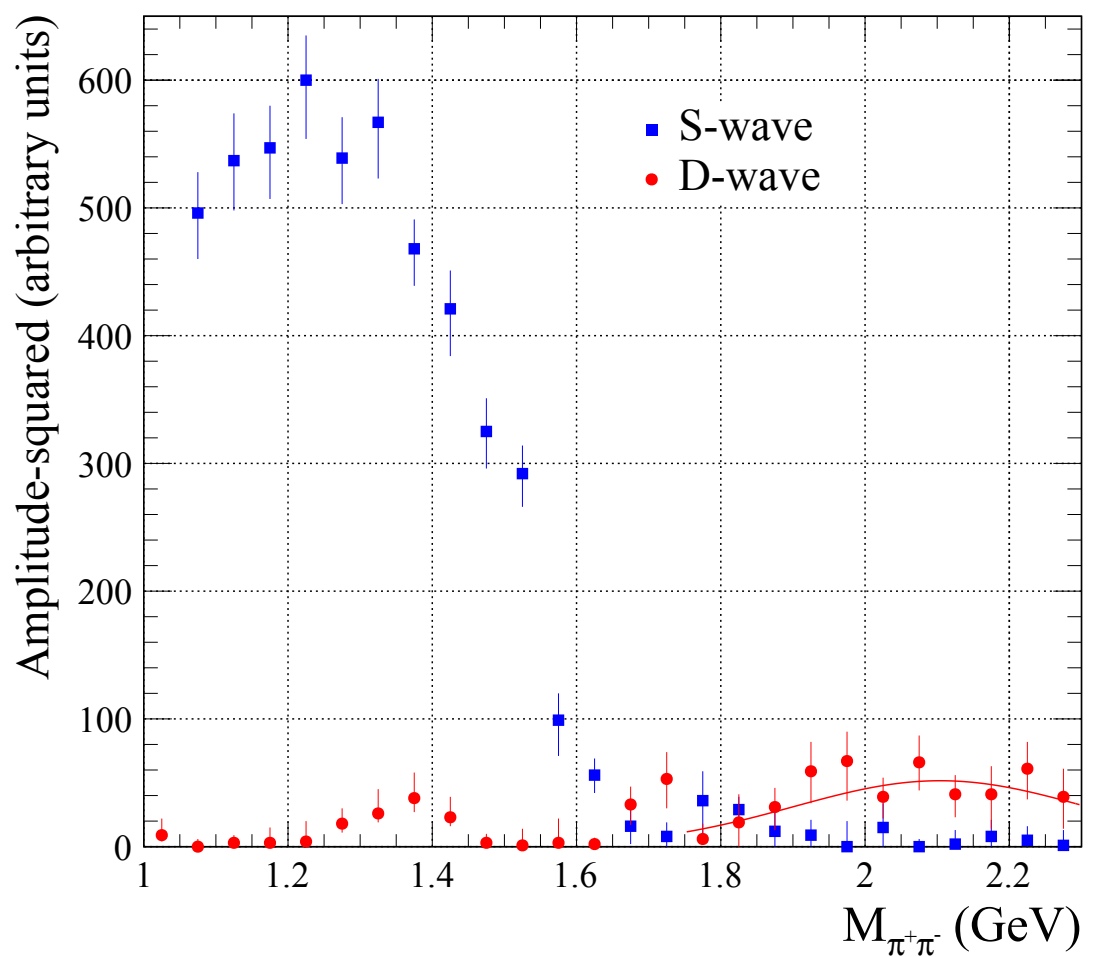

Figure 1. Extraction of tensor-Pomeron parameters from a Gaussian fit to the exclusive $\pi^{ \pm}$Axial Field Spectrometer data. The fit yields a mean mass value $\left\langle M_{\pi^{+} \pi^{-}}\right\rangle=2.10 \mathrm{GeV}$ of width $\Delta= \pm 0.68 \mathrm{GeV}$.

The D-wave dominates at masses above $\sim 2 \mathrm{GeV}$, and according to the presumed interpretation in Ref. [5] it corresponds to a spin-2 tensor glueball of mass $\mathbf{M}_{\mathrm{tgb}}$. A Gaussian fit to this enhancement 
yields $\mathrm{M}_{\mathrm{tgb}}=2.10 \pm 0.68 \mathrm{GeV}$. Identifying $\mathrm{M}_{\mathrm{tgb}}^{2}$ with the saturated glueball-like enhancement of the MBR-model parameter $s_{0}$ (see Eq. 2) yields $s_{0}=4.42 \pm 0.34 \mathrm{GeV}^{2}$. Using this value in Eq. 2 we

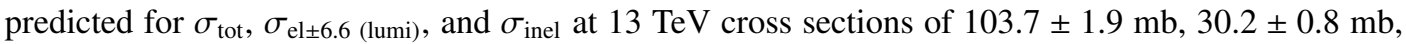
and $73.5 \pm 1.3 \mathrm{mb}$, respectively. The ATLAS- and TOTEM-measured cross sections at $\sqrt{s}=7$ and 8 $\mathrm{TeV}$ [13-15] are shown in Table 1 along with the NBR predictions.

Table 1. The total, elastic, and total inelastic MBR predictions (in mb) at $\sqrt{s}=7,8$ and $13 \mathrm{TeV}$ compared to measurements at the LHC by TOTEM and ATLAS.

\begin{tabular}{cllll}
\hline $\begin{array}{c}\sqrt{s} \\
(\mathrm{TeV})\end{array}$ & MBR Experiment & $\begin{array}{l}\sigma_{\text {tot }} \\
(\mathrm{mb})\end{array}$ & $\begin{array}{l}\sigma_{\text {el }} \\
(\mathrm{mb})\end{array}$ & $\begin{array}{l}\sigma_{\text {inel }} \\
(\mathrm{mb})\end{array}$ \\
\hline 7 & MBR & $95.4 \pm 1.2$ & $26.4 \pm 0.3$ & $69.0 \pm 1.0$ \\
& TOTEM & $98.3 \pm 2.9$ & $24.8 \pm 1.3$ & $73.7 \pm 1.7$ \\
& TOTEM_Lum_ind & $98.0 \pm 2.5$ & $25.2 \pm 1$ & $72.9 \pm 1.5$ \\
& ATLAS & $95.35 \pm 1.36$ & $24.00 \pm 0.60$ & $71.34 \pm 0.90$ \\
\hline & & & & \\
8 & MBR & $97.1 \pm 1.4$ & $27.2 \pm 0.4$ & $69.9 \pm 1.0$ \\
& TOTEM & $101.7 \pm 2.9$ & $27.1 \pm 1.4$ & $74.7 \pm 1.7$ \\
\hline & & & & \\
13 & MBR & $103.7 \pm 1.9$ & $30.2 \pm 0.8$ & $73.5 \pm 1.3$ \\
& ATLAS & xxx & xxx & $73.1 \pm 0.9(\exp ) \pm 3.8($ extr) $\pm 6.6($ lumi) \\
& CMS & xxx & xxx & $71.3 \pm 0.6(\exp ) \pm 2.7($ extr) $\pm 2.1($ lumi) \\
\hline
\end{tabular}

The ATLAS and CMS cross-section entries are imported from Refs. [7] (a) and (b), respectively. Below, we list all the ATLAS results presented in [7] (a).

1. Elastic cross section:

$$
\sigma_{\mathrm{el}}^{\mathrm{ALA}}(7 \mathrm{TeV})=24.00 \pm 0.19(\text { stat }) \pm 0.57(\text { syst }) \mathrm{mb}
$$

2. Nuclear slope:

$$
b^{\mathrm{ALFA}}(7 \mathrm{TeV})=19.73 \pm 0.14(\text { stat }) \pm 0.26 \text { (syst) } \mathrm{GeV}^{-2}
$$

3. Total cross section

$$
\sigma_{\text {tot }}^{\mathrm{ALFA}}(7 \mathrm{TeV}=95.35 \pm 0.38(\text { stat }) \pm 1.25(\exp ) \pm 0.37(\text { extr }) \mathrm{mb}
$$

4. Inelastic cross section:

- $\sigma_{\text {inel }}^{\mathrm{ALFA}}(7 \mathrm{TeV})=71.34 \pm 0.36$ (stat) \pm 0.83 (syst) $\mathrm{mb}$

- $\sigma_{\text {inel }}^{\mathrm{MBTS}}(7 \mathrm{TeV})=69.4 \pm 2.4(\exp ) \pm 6.9($ extr) $\mathrm{mb}$

- $\sigma_{\text {inel }}^{\mathrm{MBTS}}(13 \mathrm{TeV})=73.1 \pm 0.9(\exp ) \pm 3.8$ (extr) \pm 6.6 (lumi) $\mathrm{mb}$

Shown in Table 1 is also a recent measurement of the total inelastic cross section by ATLAS at $\sqrt{s}=13 \mathrm{TeV}$ [16], $\sigma_{\text {inel }}=73.1 \pm 0.9$ (exp) \pm 3.8 (extr) \pm 6.6 (lumi) $\mathrm{mb}$, which, apart from the extrapolation and luminosity uncertainties, is in excellent agreement with MBR. All the other measurements are also in good agreement with the predictions. The tensor-Pomeron-based prediction of $\sigma_{\text {inel }}$ at $\sqrt{s}=13 \mathrm{TeV}$ agrees with the ATLAS measurement at the $1 \%$ level.

It should be emphasized that the tensor-Pomeron hypothesis predicts directly only the total cross section. As discussed above, the elastic cross section for $\sqrt{s} \leq 1.8 \mathrm{TeV}$ is obtained from the global 
fit [9], while for $1.8<\sqrt{s} \leq 13 \mathrm{TeV}$ we use an extrapolation of the global-fit ratio of $\sigma_{\mathrm{el}} / \sigma_{\mathrm{tot}}$, which is slowly varying with $\sqrt{s}$, multiplied by $\sigma_{\text {tot }}$.

The total inelastic cross section is calculated as the difference between the total and the elastic cross sections. Thus, a measured lower $\sigma_{\text {el }}$ would result in a higher $\sigma_{\text {inel }}$. As seen in Table 1 , the MBR $\sigma_{\text {el }}$ is larger than the TOTEM and CMS measurements by $\sim 2 \mathrm{mb}$ at $\sqrt{s}=7 \mathrm{TeV}$, which would imply a higher MBR prediction for $\sigma_{\text {inel }}$ at $13 \mathrm{TeV}$ by $\sim 2 \mathrm{mb}$ as well. This interplay between $\sigma_{\text {el }}$ and $\sigma_{\text {inel }}$ should be carefully taken into consideration as more measurements of both $\sigma_{\text {el }}$ and $\sigma_{\text {tot }}$ at $\sqrt{s}=8 \mathrm{TeV}$ and $\sqrt{s}=13 \mathrm{TeV}$ with reduced luminosity and extrapolation uncertainties become available.

\section{Summary and conclusions}

We present the predictions of the total, elastic scattering, and total-inelastic proton-proton cross sections at $\sqrt{s}=7,8$ and $13 \mathrm{TeV}$ of the MBR model, based on a Regge-theory inspired tensor-Pomeron implementation of the RENORM model for hadronic diffraction, and compare them with experimental results by the TOTEM, ATLAS, and CMS Collaborations. All measured cross sections are in good agreement within the experimental uncertainties of the data and the theoretical uncertainties of the model, reaching down to the $\sim 1 \%$ level.

We emphasize that the tensor-Pomeron model predicts directly only to the total cross section, while the predicted $\sigma_{\mathrm{el}}$ is calculated by an extrapolation of the global-fit [9] ratio of $\sigma_{\mathrm{el}} / \sigma_{\text {tot }}$ multiplied by the tensor-Pomeron $\sigma_{\text {tot }}$ and therefore carries the larger uncertainties of this ratio. Future measurements of both $\sigma_{\mathrm{el}}$ and $\sigma_{\text {tot }}$ at $\sqrt{s}=8 \mathrm{TeV}$ and $\sqrt{s}=13 \mathrm{TeV}$ with reduced luminosity and extrapolation uncertainties are needed to clarify the current experimental status.

\section{Acknowledgments}

Warmly acknowledged are Dr. Michael Albrow for providing a copy of the thesis of Peter C. Cesil, where the tensor-Pomeron data and analysis are discussed, and Dr. Robert Ciesielski for extracting the tensor-Pomeron parameters from the data and for many invaluable discussions.

\section{References}

[1] Konstantin Goulianos, Review of RENORM Diffractive Predictions for LHC up to $8 \mathrm{TeV}$ and Extension to $13 \mathrm{TeV}$, PoS(DIS 2015)073.

[2] K. Goulianos, Hadronic diffraction: where do we stand? [arXiv:hep-ph/0407035];

K. Goulianos, Diffraction in QCD [arXiv:hep-ph/0203141].

[3] M.G. Albrow, T.D. Coughlin, J.R. Forshaw, Prog. Part. Nucl. Phys. 65, 149 (2010) [DOI:10.1016/j.ppnp.2010.06.001 [arXiv:1006.1289].

[4] M.G. Albrow, private communication, discussions about the Axial Field Spectrometer results on exclusive $\pi^{+} \pi^{-}$.

[5] Peter Charles Cesil, A Search for Centrally Produced Glueballs in Proton-Proton Interactions, $\mathrm{PhD}$ thesis, University of Cambridge, September 1984.

[6] K. Goulianos, RENORM tensor-Pomeron diffractive predictions, in 51st Rencontres de Moriond on QCD and High Energy Interactions, 19-26 Mar 2016. La Thuile, Italy C16-03-19.1 [arXiv:1606.01302v2 [hep-ph] 3 Jun 2016]. 
[7] Presented at 24th International Workshop on Deep-Inelastic Scattering and Related Subjects (DIS 2016), Hamburg, Germany, April 11-25, 2016 [https://dis2016.desy.de/]:

(a) Maciej Trzebinzki (ATLAS Collaboration), Measurements of Total, Elastic, Inelastic and Diffractive Cross Sections with the ATLAS Detector;

(b) Hans Van Haevermaet (CMS Collaboration), Measurement of the inelastic cross section at $13 \mathrm{TeV}$.

[8] R. Ciesielski and K. Goulianos, MBR Monte Carlo Simulation in PYTHIA-8 [arXiv:1205.1446].

[9] R. J. M. Covolan, J. Montanha and K. Goulianos, Phys. Lett. B 389, 176 (1996).

[10] M. Froissart, Phys. Rev. 3, 123 (1961).

[11] A. Martin, Nuovo Cimento 42, 930 (1966).

[12] A. Martin, Phys. Rev. D 80, 065013 (2009).

[13] Marek Tasevsky (ATLAS Collaboration), Diffraction and forward physics of the ATLAS experiment from Run I, Diffraction 2014, AIP Conf. Proc. 1654 (2015) 040002, (2015-04-10) [DOI:10.1063/1.4915967].

[14] G. Antchev et al. (TOTEM Collaboration), Eur. Phys. Lett. 101 (2013) 21002, Eur. Phys. Lett. 101 (2013) 21004, Eur. Phys. Lett. 101 (2013) 21003.

[15] G. Antchev et al. (TOTEM Collaboration), Phys. Rev. Lett. 111, 012001 (2013).

[16] D. Schaefer (CERN), Recent soft QCD results from the LHC, in 2016 Aspen Winter Conference in Particle Physics: Particle Physics on the Verge of Another Discovery?, 11-16 January 2016, ACP, Aspen, CO. 